\title{
THE MODULE TYPE OF A RING(1)
}

BY

W. G. LEAVITT

1. Introduction. In the following we will consider only rings with unit. It is well known that a finitely based module $\left({ }^{2}\right)$ (over some specified ring) can have bases of different lengths $\left({ }^{3}\right)$. Examples of such modules are given in $[1 ; 2 ; 3]$.

A module will be said to have dimension $n$ if it has a basis of length $n$, and if all of its bases are of the same length. A ring $A$ will be called dimensional if all finitely based $A$-modules have dimension. The class of dimensional rings includes all rings with the ascending chain condition on left ideals (left Noetherian rings) $[4$, p. 32$]$, and thus all rings with the descending chain condition (left Artinian rings) [4, p. 71]. The class also includes all commutative rings $[5$, p. 563] and all subrings (with the same unit) of a division ring [5, p. 563] (or, more generally, of an Artinian ring [6, p. 249]).

In this paper it is shown that a given ring can admit only certain characteristic types of finitely based modules, and rings are classified according to to their module type. There exists a natural ordering of such types, relative to which the type of the dimensional rings is maximal. The types are shown to form a lattice, with the lattice operations related to certain operations on rings of corresponding types. It is shown, further, that whenever there exists a unit-preserving homomorphism of $A$ into $A^{\prime}$, then the type of $A^{\prime}$ is less than or equal to that of $A$. As a corollary, we have a result (due to $\mathrm{O}$. T. O'Meara) according to which the dimensionality of $A^{\prime}$ implies that of $A$. This permits us to add substantially to the class of dimensional rings.

In the final section, it is shown that rings exist of arbitrary type. These are quotient rings of rings of noncommutative polynomials, constructed in a manner similar to that of [3]. It is shown that the rings so constructed are (Jacobson) semi-simple. An alternative construction is indicated, leading to rings of arbitrary type which are semi-simple, prime, and, except for the case of a ring which is nondimensional when regarded as a module over itself, have no zero divisors.

Presented to the Society, November 26, 1960, under the title $A$ classification of rings by module type; received by the editors May 27, 1961.

(1) Research for this paper was supported by the National Science Foundation and the University of Nebraska Research Council. The author would like to thank the referee for a number of valuable suggestions.

(2) All modules will be assumed to be unitary left modules.

( $)$ It is easily shown that this cannot happen if the module has an infinite basis. In fact, whenever a module has an infinite basis, all bases have the same cardinality. 
2. Nondimensional rings. The following theorem permits a general definition of the module type of a ring.

TheOREM 1. For any nondimensional ring $A$ there exist unique positive integers $n, k$ such that (i) any $A$-module with a basis of length $<n$ has dimension; (ii) for any $A$-module $M$ with a basis of length $\geqq n$ there exists an integer $h$, where $n \leqq h<n+k$, such that $M$ has a basis of length $r$ if and only if $r=h+m k$ for some integer $m \geqq 0$. Moreover, such an $A$-module exists for arbitrary $h$.

Proof. By hypothesis there exists at least one finitely-based $A$-module which does not have dimension. Let $n$ be the length of the shortest basis to be found among all such modules, so that by definition $n$ satisfies condition (i) of the theorem. Then consider the class of all $A$-modules having a basis of length $n$, and let $n+k$ be the length of the next shortest basis to be found among all modules of this class.

Choose an $A$-module $M$ having bases of length $n$ and $n+k$, and let $N$ be an $A$-module with a basis of length $t \geqq n$. If $t<n+k$, set $h=t$. If $t \geqq n+k$, let $N_{1}$ be the module generated by the first $n+k$ elements of the basis of $N$, and $N_{2}$ the module generated by the remaining $t-(n+k)$ elements. Then $N=N_{1} \oplus N_{2}$ and since both $M$ and $N_{1}$ have bases of length $n+k, M \cong N_{1}$. But then $N_{1}$ also has a basis of length $n$, so that $N$ has a basis of length $t-k$. By repetitions of this process, it is clear that $N$ has a basis of length $h$ for some $h$ in the range $n \leqq h<n+k$. Then by reversing the argument, $N$ has a basis of length $h+m k$ for any $m \geqq 0$.

Now suppose $N$ has a basis of length $h^{\prime} \neq h+m k$. By the above process we can as well assume that $n \leqq h^{\prime}<n+k$ with $h^{\prime} \neq h$, say $h<h^{\prime}$. Let $d$ be the G.C.D. of $k$ and $h^{\prime}-h$, then $d=u\left(h^{\prime}-h\right)+v k$ for some integers $u$ and $v$. Suppose $u>0$ (if $u<0$ then $v>0$ and the proof is similar). Since $h<n+k$ we can, as above, write $M=M_{1} \oplus M_{2}$ where $M_{1}$ has a basis of length $h$. Thus $M_{1} \cong N$ and so also has a basis of length $h^{\prime}$. It follows that $M$ has a basis of length $n+k+\left(h^{\prime}-h\right)$. Continuing, $M$ has a basis of length $n+k+u\left(h^{\prime}-h\right)$, and, by using $M$ itself, in a similar way, a basis of length $n+u\left(h^{\prime}-h\right)+v k$. Since $d<k$ this violates the minimality of $n+k$, and we conclude that $N$ can have no bases other than those of length $h+m k$.

Finally, we may take the direct sum of $M$ with any number of copies of $A$, so that such an $A$-module exists for arbitrary $h$.

Remark that the above process provides an easy proof that Noetherian and Artinian rings are dimensional. Let $M$ have bases of length $n$ and $n+k$, so that $M=M_{1} \oplus M^{\prime}$ where $M^{\prime} \cong M$. But then $M^{\prime}$ also has a basis of length $n+k$, so again $M^{\prime}=M_{2} \oplus M^{\prime \prime}$ where $M^{\prime \prime} \cong M$. The process evidently yields chains $M_{1} \subset M_{1} \oplus M_{2} \subset \cdots$ and $M \supset M^{\prime} \supset M^{\prime \prime} \supset \cdots$, violating both chain conditions for $A$-modules. Since chain conditions on left ideals of $A$ imply corresponding conditions on finitely generated $A$-modules $[4, \mathrm{p} .32]$, the result follows. 
Theorem 1 permits the following classification of all rings with unit: (i) dimensional rings, type $d$; (ii) nondimensional rings, type $(n, k)$, where $(n, k)$ are the integers of Theorem 1 ; (iii) the ring with zero as its only element, type 0 .

The ring types may be partially ordered as follows: (i) $0<(n, k)<d$ for all $(n, k)$; (ii) $\left(n^{\prime}, k^{\prime}\right) \leqq(n, k)$ if and only if $n^{\prime} \leqq n$ and $k^{\prime} \mid k$. Note that the ring types form a distributive lattice under the operations

$$
(n, k) \cap\left(n^{\prime}, k^{\prime}\right)=\left(\min \left(n, n^{\prime}\right), \text { G.C.D. }\left(k, k^{\prime}\right)\right)
$$

and

$$
(n, k) \cup\left(n^{\prime}, k^{\prime}\right)=\left(\max \left(n, n^{\prime}\right), \text { L.C.M. }\left(k, k^{\prime}\right)\right) .
$$

It is well known (and easily shown) that there exists an $A$-module with bases of length $n$ and $n+k$ if and only if there exist $n+k$ by $n$ and $n$ by $n+k$ matrices $X$ and $Y$ over $A$ such that $X Y=I_{n+k}$ and $Y X=I_{n}$, where $I_{n+k}$ and $I_{n}$ are identity matrices. Note that this criterion for the existence of an $A$ module with bases of length $n$ and $n+k$ is right-left symmetric. Thus if we had introduced the concept of a "right" type of a ring, using right modules in a manner analogous to our use of left modules, the result would have been no different. In defining the type of a ring, we are therefore justified in omitting reference to the orientation of the modules used.

TheоReм 2. Let $A$ and $A^{\prime}$ be rings of type $a$ and $a^{\prime}$ respectively. If $A \rightarrow A^{\prime}$ is a unit-preserving homomorphism of $A$ into $A^{\prime}$, then $a^{\prime} \leqq a$.

Proof. If $a=d$ or if $A^{\prime}=0$ the theorem is obvious. Since $A \rightarrow A^{\prime}$ is unitpreserving, the existence of matrices over $A$ satisfying the conditions of the above remark would imply the existence of matrices over $A^{\prime}$ satisfying the same conditions. Hence $a^{\prime}=d$ implies $a=d$. Moreover, if $a=(n, k)$ then $A^{\prime}$ admits a module with bases of length $n$ and $n+k$. From Theorem 1 it then follows immediately that $n^{\prime} \leqq n$ and $k^{\prime} \mid k$.

Corollary 1( $\left.{ }^{4}\right)$. A ring $A$ is dimensional if and only if there exists a unitpreserving homomorphism of $A$ into a dimensional ring $A^{\prime}$.

Corollary 2. A subring (with the same unit) of a dimensional ring is dimensional.

Proof. The homomorphism is the natural inclusion map. More generally, if $A$ has a subring which shares its unit with $A$, then the type of $A$ is less than or equal to that of the subring.

Corollary 3. A commutative ring is dimensional.

Proof. By Zorn's lemma, any ring with unit has a maximal ideal. Let $J$

( ${ }^{`}$ Corollary 1 (with a different proof), and Corollaries 2 and 3, are due to O. T. O'Meara (unpublished). 
be a maximal ideal of the commutative ring $A$, then the homomorphism is the natural map $A \rightarrow A / J$ onto the field $A / J$. Note that an onto map is automatically unit-preserving.

COROLlaRy 4. Let $\left\{x_{i}\right\}_{i \in I}$ be a set of symbols, where $I$ is any index set. If $A$ is a ring of type $a$, then the ring $B$ of polynomials in (commuting or noncommuting) symbols $\left\{x_{i}\right\}$ over $A$ is also of type $a$.

Proof. This is clear, for the map of polynomials to their constant terms is a homomorphism of $B$ onto $A$, while the natural inclusion map is a unitpreserving homomorphism of $A$ into $B$.

Remark that the necessity of the requirement that the homomorphism be unit-preserving is shown by the example of the direct sum of two rings of different types. Homomorphisms exist both ways, but in this case the inclusion map is not unit-preserving.

Corollary 5. Let $A$ be a ring of type a. A right (left) ideal of $A$ which is a ring with unit is of type

Proof. Let $I$ be a right ideal of $A$, with unit $e$. For any $x \in A$, exe $=e x$, so the mapping $A \rightarrow I$ defined by $x \rightarrow e x$ is a homomorphism of $A$ onto $I$.

THEOREM 3. If $A_{1}$ and $A_{2}$ are rings of type $a_{1}$ and respectively, then $A=A_{1} \oplus A_{2}$ is of type $a_{1} \cup a_{2}$.

Proof. Let $A$ be of type $a$, then the natural homomorphisms of $A$ onto $A_{1}$ and $A_{2}$ require, by Theorem 2 , that $a_{1} \leqq a$ and $a_{2} \leqq a$ so $a_{1} \cup a_{2} \leqq a$. Thus if either $a_{1}=d$ or $a_{2}=d$, then $d=a=a_{1} \cup a_{2}$. The theorem is also obvious if either $A_{1}=0$ or $A_{2}=0$. Thus suppose $a_{1}=\left(n_{1}, k_{1}\right)$ and $a_{2}=\left(n_{2}, k_{2}\right)$ and $a_{1} \cup a_{2}=(n, k)$. Since $a_{1} \leqq a_{1} \cup a_{2}$, we have $n_{1} \leqq n$ and so by Theorem 1 there exists an $A_{1}$ module $\bar{M}_{1}$ with bases of length $n$ and $n+m k_{1}$ for arbitrary $m$. Since $k_{1} \mid k_{\text {: }}$ we may choose $m$ so that $M_{1}$ has bases of length $n$ and $n+k$. Similarly there exists an $A_{2}$-module $M_{2}$ with bases of length $n$ and $n+k$. Defining $A_{1} M_{2}=0$ and $A_{2} M_{1}=0$, then $M_{1} \oplus M_{2}$ is an $A$-module with bases of length $n$ and $n+k$. Thus $a \leqq(n, k)$ and so $a=a_{1} \cup a_{2}$.

THEOREM 4. If $A_{1}$ and $A_{2}$ are rings of type $a_{1}$ and $a_{2}$ respectively, then $A_{1} \otimes A_{2}$ is of type $\leqq a_{1} \cap a_{2}$.

Proof. This is clear, since there exist unit-preserving homomorphisms of $A_{1}$ and $A_{2}$ into $A_{1} \otimes A_{2}$, namely $A_{1} \rightarrow A_{1} \otimes 1$ and $A_{2} \rightarrow 1 \otimes A_{2}$.

Remark that the question is open as to whether or not rings $A_{1}$ and $A_{2}$ exist for which the type of $A_{1} \otimes A_{2}$ is actually less than $a_{1} \cap a_{2}$. The broader problem of finding a construction, from given rings $A_{1}$ and $A_{2}$, of a ring of type equal to $a_{1} \cap a_{2}$ is therefore also open.

Theorem 5. If $A$ is a ring of type a, then the complete matrix ring $A_{m}$ is 
(i) of type $d$ if $a=d$; (ii) if $a=(n, k)$, of type $((n+r) / m, k / g)$, where $g=$ G.C.D. $(k, m)$ and $r$ is the least non-negative integer such that $m \mid n+r$.

Proof. Let $A_{m}$ be of type $b$. Since there exists a unit-preserving homomorphism $A \rightarrow A_{m}$, it follows that $b \leqq a$, and so $b=d$ only if $a=d$. Thus suppose $b=(p, h)$. Then there must exist $p+h$ by $p$ and $p$ by $p+h$ matrices over $A_{m}$ whose products are identity matrices. But if we regard the elements of these matrices as submatrices of matrices over $A$, this means that there exist $m(p+h)$ by $m p$ and $m p$ by $m(p+h)$ matrices whose products are identity matrices over $A$. Thus $A$ is not dimensional and so assertion (i) is proved. Writing $a=(n, k)$ this also shows, by Theorem 1 , that $m p \geqq n$ and $k \mid m h$. Hence $k / g \mid h$, where $g=$ G.C.D. $(k, m)$.

Now by Theorem 1 there exists an $A$-module with bases of length $n+r$ and $n+r+s k$ for arbitrary non-negative $r$ and $s$. Choose the least $r \geqq 0$ such that $m \mid n+r$ and choose $s=m / g$. The matrices relative to these bases may be partitioned into $m$ by $m$ submatrices furnishing matrices over $A_{m}$ of sizes $(n+r) / m$ and $(n+r) / m+k / g$, whose products are identity matrices over $A_{m}$. Thus $p \leqq(n+r) / m$ and $h \mid k / g$. But then $h=k / g$, and since $m p \geqq n$, it follows that $p=(n+r) / m$.

TheOREM 6. If $D$ is a division ring, then any subring of $D_{m}$ is dimensional.

Proof. Let $e$ be the unit of the subring $A$ of $D_{m}$. We may regard $D_{m}$ as the set of linear transformations of an $m$-dimensional vector space, and choose its basis $\left\{\alpha_{i}\right\}, i=1, \cdots, m$, so that $\left\{\alpha_{j+1}, \cdots, \alpha_{m}\right\}$ is the basis of the nullspace of $e$ (that is, we are considering the ring isomorphic to $A$ under an appropriate inner automorphism of $D_{m}$ ). Regarding $e$ as restricted to the subspace $S_{j}$ (with basis $\left\{\alpha_{i}\right\}, i=1, \cdots, j$ ), $e$ is nonsingular and since it is idempotent, $e=I_{j}$. Since $x e=x$ for all $x \in A$, we may also regard $x$ as restricted to $S_{j}$, that is, $A$ is a subring (with the same unit) of $D_{j}$. But by Theorem $5, D_{j}$ is dimensional and thus by Corollary 2, $A$ is also dimensional.

Corollary 6. Any locally matrix ring is dimensional.

Proof. If $A$ is nondimensional, there exist matrices $X, Y$ such that $X Y=I_{n+k}, Y X=I_{n}$ for some $n, k>0$. But such matrices are impossible in a locally matrix ring, since the set of all elements of $X$ and $Y$ (together with the unit) generate a dimensional ring, namely a subring of some $D_{m}$.

Corollary 7. If $A$ has a maximal ideal which does not contain the socle of $A$, then $A$ is dimensional.

Proof. Let $I$ be the maximal ideal of $A$ which does not contain some minimal one-sided ideal of $A$. Then $A / I$ is a simple ring with a minimal ideal and so $[7$, p. 90] locally matrix. By Corollaries 1 and $6, A$ is dimensional. Alternatively, by $[7$, p. 88], $A / I$ is left Artinian and hence dimensional, so the result again follows from Corollary 1. 
TheOREM 7. If $A$ is a ring with pivotal monomial( $\left.{ }^{5}\right)$, then $A$ is dimensional.

Proof. Let $I$ be any maximal ideal of $A$. Then $A / I$ is simple with unit and hence $[7$, p. 4] primitive. Since the property of having a pivotal monomial is preserved under homomorphism, by $[8, \mathrm{p} .357] A / I$ is isomorphic with some $D_{m}$. The result then follows from Theorem 5 and Corollary 1 .

\section{COROLlaRy 8. Every PI ring and every algebraic ring of bounded degree is dimensional.}

Proof. By [8, p. 353] any such ring has a pivotal monomial.

Remark that the converse of Theorem 7 does not hold, as is shown by the example of the polynomial ring, say over the rational field, in two noncommutative variables. By Corollary 4 this ring is dimensional, but certainly cannot have a pivotal monomial.

3. The existence of rings of arbitrary type. The ring constructed by Everett [1] is of type $(1,1)$, while the rings constructed in $[2 ; 3]$ are of type $(n, 1)$ for arbitrary $n$. It is the purpose of the present section to construct a ring of type $(n, k)$ for arbitrary $n$ and $k$. Note that $(n, 1) \cup(1, k)=(n, k)$, so that since rings of type $(n, 1)$ have already been constructed in [2] and [3], it suffices by Theorem 3 to construct rings of type $(1, k)$ for arbitrary $k \geqq 2$.

Consider the ring $R$ of polynomials (free algebra) with unit generated, over the field of integers modulo 2 , by non-commutative symbols $\left\{x_{i}, y_{j}\right\}$, $i, j=1, \cdots, k+1$. Form column and row matrices $X=\left(x_{1} \cdots x_{k+1}\right)^{\prime}$ and $Y=\left(y_{1} \cdots y_{k+1}\right)$. Let $H$ be the two-sided ideal generated by the elements of $X Y-I_{k+1}$ and $Y X-1$, where $I_{k+1}$ is the $k+1$ by $k+1$ identity matrix, and let $A=R / H$. It is clear (see [2 or 3] for details) that in each residue class modulo $H$ there exists a unique polynomial none of whose terms contains an occurrence of any of the set $\left\{x_{i} y_{j}, y_{1} x_{1}\right\}$. Thus $A$ may be regarded as the ring of all such polynomials, provided multiplication is modified by requiring that, in any product $x_{i} y_{j}$ is to be replaced by $\delta_{i j}$ (where $\delta_{i j}=1$ if $i=j$, zero otherwise), and $y_{1} x_{1}$ by $\sum_{2}^{k+1} y_{i} x_{i}+1$. From this it follows that all monomials of $A$ are of form $u v$ where $u$ is a product of $y$ 's (or $u=1$ ) and $v$ a product of $x$ 's $($ or $v=1)$.

Clearly there exists an $A$-module with bases of length 1 and $k+1$, namely the ring $A$ itself, regarded as an $A$-module. Thus it suffices to show that the existence of $q$ by 1 and 1 by $q$ matrices $\Phi, \Theta$ satisfying

$$
\Phi \Theta=I_{q}, \quad \Theta \Phi=1, \quad \text { for some } q \not \equiv 1(\bmod k),
$$

leads to a contradiction. Note that if $\Psi$ is an arbitrary $q$ by $q$ unimodular matrix, then $\Theta \Psi$ and $\Psi^{-1} \Phi$ also satisfy (1).

Define the degree $d[u]$ of a monomial $u$ as the length of $u$ (or 0 if $u=1$ ),

${ }^{(5)}$ For the definition of pivotal monomial see [8, p. 352]. The possibility that this theorem might be true was suggested by M. P. Drazin. 
and for an arbitrary $\alpha$ of $A$, the degree $d[\alpha]=\max \{d[u]\}$ for the terms of $\alpha$. Terms of $\alpha$ of degree equal to $d[\alpha]$ will be called longest terms of $\alpha$. We define an ordering in $A$ as follows: (i) For all values of the indicated subscripts, $y_{j}<x_{i}, x_{i-1}<x_{i}, y_{j-1}<y_{j}$. (ii) If $u$ and $v$ are monomials, $u<v$ if $d[u]<d[v]$, and if $d[u]=d[v]$, then $u<v$ if $u$ precedes $v$ lexicographically. (iii) Rearrange the terms of polynomials to read in descending order (that is, if $\alpha=w_{1}+w_{2}+\cdots$, then $w_{1}>w_{2}>\cdots$; note that we will call $w_{1}$ the leading term of $\alpha$ ). Then $\alpha<\beta$ if $\alpha$ precedes $\beta$ lexicographically. Also $0<\alpha$ for all $\alpha \neq 0$.

A set $\left\{\alpha_{i} \beta_{i}\right\}, i=1, \cdots, h$, is said to be diminished if it is replaced by a set $\left\{\alpha_{i 1} \beta_{i 1}\right\}, i=1, \cdots, h_{1} \equiv h(\bmod k)$ satisfying one of the following: (i) $\max \left\{d\left[\alpha_{i 1}\right]+d\left[\beta_{i 1}\right]\right\}<\max \left\{d\left[\alpha_{i}\right]+d\left[\beta_{i}\right]\right\}$; (ii) the maxima are equal, but there are fewer $i$ for which $d\left[\alpha_{i 1}\right]+d\left[\beta_{i 1}\right]$ equals the maximum; (iii) $h_{1}=h$, the maxima and the number of $i$ for which the maximum is attained equal, but $\alpha_{i 1}<\alpha_{i}$ for some $i$, while $\alpha_{j 1} \leqq \alpha_{j}$ for all $j \neq i$.

Now suppose it is always possible to replace $\Theta$ and $\Phi$ by $\Theta_{1}$ and $\Phi_{1}$ satisfying (1) such that $\left\{\theta_{i}, \phi_{i}\right\}$ is diminished when replaced by $\left\{\theta_{i 1} \phi_{i 1}\right\}$, then the proof is immediate. For eventually an element of zero degree, namely 1 , must appear, say in $\Theta$. But then an elementary matrix $\Psi$ produces $\Theta \Psi$ $=(1,0, \cdots, 0)$, and clearly $\Theta \Psi, \Psi^{-1} \Phi$ could not satisfy (1). It will accordingly be assumed that for the matrices $\Theta, \Phi$ satisfying (1) the set $\left\{\theta_{i} \phi_{i}\right\}$ is minimal (in the sense that it cannot be diminished in the manner indicated above), and a transformation which, if it existed, would give a diminished set, is said to violate minimality.

In the following we will use the designation $u \in \alpha$ to mean that $u$ is a term of $\alpha$. Let $u \in \theta_{i}$ and $v \in \phi_{i}$ for some $i$. We call $u v$ a reaction if it must be reduced (that is, if either (i) $u$ ends in $y_{1}$ and $v$ begins with $x_{1}$, or (ii) $u$ ends in some $x_{i}$ and $v$ begins with some $y_{j}$ ). A term is bound in $\theta_{i} \phi_{i}$ if it results from some reaction in $\theta_{i} \phi_{i}$ and has degree equal to the sum of the degrees of the reacting terms. Terms of lesser degree resulting from a reaction will be called secondary reaction terms. All other terms of $\theta_{i} \phi_{i}$ are said to be unbound in $\theta_{i} \phi_{i}$. A term is bound in a range $a<i \leqq b$ if it is bound in some $\theta_{i} \phi_{i}$ for $i$ in the range. Terms are unbound in the range if they are unbound in all $\theta_{i} \phi_{i}$ of the range. A product $\alpha \beta$ is said to be full if $d[\alpha \beta]=d[\alpha]+d[\beta]$ and almost full if $d[\alpha \beta]=d[\alpha]+d[\beta]-1$.

Lemma 1. (i) $A$ necessary and sufficient condition for $\alpha \beta$ to be full is that a longest term of $\alpha$ end in some $y_{j}$ or a longest term of $\beta$ begin with some $x_{i}$. (ii) An $\alpha \beta$ which is not full is almost full if and only if a term of $\alpha$ of degree $d[\alpha]-1$ ends in some $y_{j}$ or a term of $\beta$ of degree $d[\beta]-1$ begins with some $x_{i}$.

Proof. The necessity of (i) is obvious. Thus suppose there exist longest terms $u \in \alpha, v \in \beta$ with either (or both) $u$ ending in some $y_{j}$ and $v$ beginning with some $x_{i}$. If $u v$ is unbound in $\alpha \beta$, then clearly, being of maximal degree, it cannot be cancelled by any other term of $\alpha \beta$. But if $u v$ is bound in $\alpha \beta$, it 
must be bound to a reaction in $\alpha \beta$, or if $u v$ is itself a reaction whose results are to be cancelled, there must be corresponding terms bound to it. In either case there would be cross-product terms which would be unbound, and hence uncancelled.

To establish (ii), note that if all the longest terms of $\alpha$ end in some $x_{i}$ and all longest terms of $\beta$ begin with some $y_{j}$, then for $\alpha \beta$ to be almost full we must have $u \in \alpha$ with $d[u]=d[\alpha]-1$ ending in some $y_{j}$ or $v \in \beta$ with $d[v]$ $=d[\beta]-1$ beginning with some $x_{i}$. The products of such terms with, respectively, a longest term of $\alpha$ or a longest term of $\beta$ could not cancel each other, are unbound, and are of maximal degree. Thus, as in the previous paragraph, they would be uncancelled.

Remark that by using the proper elementary matrix $\Psi$ it is possible to obtain $\Theta \Psi$ in which $\theta_{h}$ has been replaced by $\theta_{h}+\sum_{i \neq h} \theta_{i} \alpha_{i}$ for arbitrary $\alpha_{i}$, while all other elements are unchanged. However, in so doing $\Phi$ must be replaced by $\Psi^{-1} \Phi$ whose $i$ th element is $\phi_{i}+\alpha_{i} \phi_{h}$. We will also agree, by using permutation matrices, that we have rearranged so that $\theta_{i} \phi_{i}>\theta_{i+1} \phi_{i+1}$, $i=1,2, \cdots, q-1$.

Now let $N=\max \left\{d\left[\theta_{i}\right]+d\left[\phi_{i}\right]\right\}$. We separate all $\theta_{i} \phi_{i}$ into the following classes: Class $\mathrm{A}, d\left[\theta_{i} \phi_{i}\right]=N$; Class $\mathrm{B}, d\left[\theta_{i}\right]+d\left[\phi_{i}\right]=N$ and $\theta_{i} \phi_{i}$ almost full; Class C, $d\left[\theta_{i} \phi_{i}\right]=N-1$ and $\theta_{i} \phi_{i}$ full; Class $\mathrm{D}, d\left[\theta_{i} \phi_{i}\right] \leqq N-2$.

LeMma 2. Let $v$ be a monomial for which $\theta v$ is full or almost full, and let $w$ be the leading term of $\theta v$. (i) If $v$ begins with some $y_{j}$ then $w=u v$ where $u$ is the first term of $\theta$ which ends with some $y_{h}$. (ii) If $v$ begins with some $x_{i}$ and $u$ is the leading term of $\theta$, then $w=u v$ (or is bound to $u v$ if $u v$ is a reaction) in all but the exceptional case: $u$, $v$ have form $u=u^{\prime} y_{1}, v=x_{1} v^{\prime}$ with also $u^{\prime} y_{2} \in \theta$. In this case $w=u^{\prime} y_{2} x_{1} v^{\prime}$.

Proof. This is clear from the way monomials were ordered. Remark that in (ii) $\theta v$ is automatically full.

Note 1. From this lemma (and the ordering) it is clear that if $v$ and $z$ begin with the same symbol, and $\theta v$ is full or almost full, then if $u_{1} v_{1}$ is the leading term of $\theta v$, the leading term of $\theta z$ is $u_{1} z_{1}$ where $z_{1}$ agrees with $z$ except that its initial symbol is the same as that of $v_{1}$. If, for example, $z$ is an initial segment of $v$, then $u_{1} z_{1}$ is an initial segment of $u_{1} v_{1}$.

Lemma 3. If $v>z$ and either $\theta v$ is full or both $\theta v$ and $\theta z$ are almost full, then the leading term of $\theta v$ exceeds the leading term of $\theta z$ in all but the case: $v, z$ have form $v=x_{1} v^{\prime}, z=x_{j} z^{\prime}, j \neq 1$, with $d[v]=d[z]$, and some $u^{\prime} y_{1}$ leads in $\theta$. In the exceptional case the inequality is reversed.

Proof. If $d[v]>d[z]$ and $\theta v$ is full, or both $\theta v$ and $\theta z$ are almost full, then the degree of the leading term of $\theta v$ exceeds that of $\theta z$. Thus suppose $d[v]$ $=d[z]$. If $v$ begins with some $y_{j}$, then by the ordering of monomials $z$ must begin with some $y_{h}$, and the result follows from (i) of Lemma 2. Also, if $v$ 
begins with some $x_{i}$ the result is clear from the ordering, for only in the exceptional case will $\theta z$ have a term exceeding the leading term of $\theta v$, namely $u^{\prime} y_{1} x_{j} z^{\prime}>u^{\prime} y_{2} x_{2} v^{\prime}$.

Remark that from this lemma when $\theta \phi$ is full, its leading term is the leading term of $\theta v$ for some $v \in \phi$. Moreover, in all but the exceptional case, $v$ is the leading term of $\phi$. Also note that if $\theta \phi$ is almost full, then its leading term is $u v$, where either $u$ leads in $\theta$ and $v$ is the first term of $\phi$ beginning with some $x_{i}$, or $u$ is the first term of $\theta$ ending in some $y_{j}$ and $v$ leads in $\phi$. In either case $u v$ is the leading term of $\theta v$ for some $v \in \phi$.

LEMMA 4. Let $w=u v$ or a term bound to $u v$ (if $u v$ is a reaction). If $w$ is the leading term of $\theta v_{2}$, where $\theta v_{2}$ is full or almost full and $d\left[v_{2}\right]>d[v]$, then $u$ is the leading term of $\theta z$ for some monomial $z$.

Proof. Consider, first the case $u v$ is not a reaction, so that $w=u v$. Since $d\left[v_{2}\right]>d[v]$, it is clear that $v$ must be identical with a final segment of $v_{2}$. Thus, as in Note $1, u$ must be the leading term of $\theta v_{3}$ for $v_{3}$ an initial segment of $v_{2}$.

If $u v$ is a reaction to which $w=u_{1} v_{1}$ is bound, then again, by Note $1, u_{1}$ is the leading term of $\theta v_{3}$ for $v_{3}$ an initial segment of $v_{2}$. But $u_{1}$ differs from $u$ only in its final symbol, so if $v_{4}$ is obtained from $v_{3}$ by a similar alteration, then $u$ is the leading term of $\theta v_{4}$.

Lemma 5. Let $w$ be the leading term of $\theta_{i} v_{2}$ for some $v_{2} \in \phi_{i}$, where $\theta_{i} v_{2}$ is full when $\theta_{i} \phi_{i}$ is full, and may be either full or almost full when $\theta_{i} \phi_{i}$ is almost full. For no $j$ can w equal (or be bound to) $u v$, where $u \in \theta_{j}, v \in \phi_{j}, d\left[v_{2}\right]>d[v]$, and $d[u]+d\left[\phi_{j}\right] \leqq d\left[\theta_{i}\right]+d\left[\phi_{i}\right]\left(\leqq d\left[\theta_{i} \phi_{i}\right]\right.$ when $\theta_{i} v_{2}$ is almost full $)$.

Proof. Suppose such $j$ exists, then by Lemma $4 u$ is the leading term of $\theta_{i} z$ for some $z$. Thus the elementary transformation $\theta_{j} \rightarrow \theta_{j 1}=\theta_{j}+\theta_{i} z$ removes $u$ while (possibly) substituting terms $<u$. Thus $\theta_{j 1}<\theta_{j}$, that is, the transformation diminishes $\theta_{j}$. Now $d[u]=d\left[\theta_{i}\right]+d[z]$ (or $d\left[\theta_{i}\right]+d[z]-1$ if $\theta_{i} v_{2}$ is almost full). Thus by hypothesis $d[z]+d\left[\phi_{j}\right] \leqq d\left[\phi_{i}\right]$. Hence this transformation, which also sends $\phi_{i} \rightarrow \phi_{i 1}=\phi_{i}+z \phi_{j}$ is such that $d\left[\theta_{i}\right]+d\left[\phi_{i 1}\right] \leqq d\left[\theta_{i}\right]+d\left[\phi_{i}\right]$. Thus the set $\left\{\theta_{i} \phi_{i}\right\}$ would be diminished, violating minimality.

Note 2. Suppose $u \in \theta_{j}, v \in \phi_{j}$. Let $w=u v$ (or $w=u_{1} v_{1}$, a term bound to $u v$ if it is a reaction). Let $t=u z$ for some $z$ with $d[z]=d[v]$ (or $t=u_{1} z_{1}$ for some $z_{1}$ with $\left.d\left[z_{1}\right]=d\left[v_{1}\right]\right)$. Assume that for some $i$ for which $d\left[\theta_{i}\right]<d[u]$ and $d[u]+d\left[\phi_{j}\right] \leqq d\left[\theta_{i}\right]+d\left[\phi_{i}\right]$, all terms of $\theta_{i} \phi_{i} \leqq t$, then $w \notin \theta_{i} v_{2}$ for any $v_{2}$ for which $\theta_{i} v_{2}$ is full. If $\theta_{i} v_{2}$ is almost full, the same statement can be made, if $d\left[\theta_{i}\right]$ is replaced by $d\left[\theta_{i}\right]-1$. This is clear from Lemma 5 , for if $u v \in \theta_{i} v_{2}$ then $d\left[v_{2}\right]>d[v]$ and also $v$ (or $v_{1}$ ) must coincide with a final segment of $v_{2}$. Thus a term of $\theta_{i} v_{2}$ could exceed $w$ only if its initial segment exceeds $u$ (or $u_{1}$ ). But such a term would exceed $t$. Thus $w$ would be the leading term of $\theta_{i} v_{2}$ contrary to Lemma 5. 
LeMmA 6. Let $w=u v$, and suppose $\left\{x_{i} v^{\prime}\right\}, i=1, \cdots, k+1$, is some set of monomials such that $d\left[x_{i} v^{\prime}\right]>d[v]$. If $\sum_{i=1}^{k+1} \alpha_{i} x_{i} v^{\prime}$ has $w$ as its leading term and if $w$ ends in $x_{j} v^{\prime}$ for some $j$, then $u$ is the leading term of $\sum \alpha_{i} z_{i}$ for some set of monomials $\left\{z_{i}\right\}$.

Proof. This is clearly similar to Lemma 4 , for we must have $v^{\prime}=v_{1} v$ for some monomial $v_{1}$ (possibly 1 ). Thus by Note $1, u$ is the leading term of $\sum \alpha_{i} x_{i} v_{1}$.

Remark that in the situation of this lemma $w$ could not be bound to a reaction $u v$, for then $w=u_{1} v_{1}$ where $u_{1}$ ends in some $y_{h}$. But $w$ has a final segment $x_{j} v^{\prime}$ of degree $>d\left[v_{1}\right]=d[v]$ which is a product of the $\left\{x_{i}\right\}$ alone.

LEMMA 7. Let $\left\{\theta_{c+i}\right\}, i=1, \cdots, k+1$ be such that $w$ is the leading term of $\sum \theta_{c+i} x_{i} v^{\prime}$, where $w$ ends in some $x_{h} v^{\prime}$, then w cannot equal uv where $u \in \theta_{j}$, $v \in \phi_{j}, d\left[x_{i} v^{\prime}\right]>d[v]$ and $d\left[v^{\prime}\right]-d[v]<d\left[\phi_{c+i}\right]-d\left[\phi_{j}\right]$, for all $i=1, \cdots, k+1$.

Proof. Suppose the contrary. As in the preceding remark, we need not consider the case $u v$ a reaction. Now from Lemma $6, u$ is the leading term of $\sum \theta_{c+i} z_{i}$ where all the $z_{i}$ have dimension $d\left[x_{i} v^{\prime}\right]-d[v]$. Consider the elementary transformation

$$
\begin{aligned}
\theta_{j} & \rightarrow \theta_{j 1}=\theta_{j}+\sum \theta_{c+i} z_{i}, \\
\phi_{c+i} \rightarrow \phi_{c+i 1} & =\phi_{c+i}+z_{i} \phi_{j}, \quad i=1, \cdots, k+1 .
\end{aligned}
$$

This transformation diminishes $\theta_{j}$, by removing $u$, and by hypothesis, $d\left[v^{\prime}\right]$ $+d\left[\phi_{j}\right]<d[v]+d\left[\phi_{c+i}\right]$. Thus $d\left[z_{i}\right]+d\left[\phi_{j}\right] \leqq d\left[\phi_{c+i}\right]$, so $d\left[\phi_{c+i 1}\right] \leqq d\left[\phi_{c+i}\right]$, and minimality is violated.

Note 3. Suppose $w=u v$ where $u \in \theta_{j}, v \in \phi_{j}$ and $t=u z$ for some $z$ with $d[z]=d[v]$. Assume that for some $v^{\prime}$ we have $x_{i} v^{\prime} \in \phi_{c+i}, i=1, \cdots, k+1$, where $d\left[x_{i} v^{\prime}\right]>d[v]$ and $d\left[v^{\prime}\right]-d[v]<d\left[\phi_{c+i}\right]-d\left[\phi_{j}\right]$ for all $i=1, \cdots, k+1$. If all terms of $\sum \theta_{c+i} \phi_{c+i} \leqq t$ and at least one ends in some $x_{h} v^{\prime}$, then $w \notin \sum \theta_{c+i} x_{i} v^{\prime}$. Suppose the contrary, then the final segment of $v^{\prime}$ must agree with $v$. Thus a term exceeding $w$ would have an initial segment $>u$, so would exceed $t$. Thus $w$ would lead in $\sum \theta_{c+i} x_{i} v^{\prime}$, contrary to Lemma 7 .

In the following we will write $\bar{\theta}_{i}$ for the sum of all longest terms of $\theta_{i}$, and $\bar{\theta}_{i}^{*}$ for all terms of degree $d\left[\theta_{i}\right]-1$; similarly $\bar{\phi}_{i}$ and $\bar{\phi}_{i}^{*}$.

LemMa 8. The set of all $\left\{\bar{\phi}_{i}\right\}$ is independent relative to the base field.

Proof. Suppose the contrary. Clearly no generality is lost in assuming there is a linear relation connecting a set $\left\{\Phi_{i_{j}}\right\}$ all of the same degree. Choose $\boldsymbol{\phi}_{h}$ from this set in such a way that $d\left[\theta_{h}\right] \leqq d\left[\theta_{i_{j}}\right]$ for all $i_{j}$ in the set, and write $\phi_{h}=\sum_{i_{j \neq h}} \phi_{i_{j}}$. Consider the elementary transformation $\phi_{h} \rightarrow \phi_{h 1}=\phi_{h}+\sum \phi_{i_{j}}$, $\theta_{i_{j}} \rightarrow \theta_{i_{j} 1}=\theta_{i_{j}}+\theta_{h}$, for all $i_{j} \neq h$ in the set. Since this removes from $\phi_{h}$ all terms of maximal degree, $d\left[\theta_{h}\right]+d\left[\phi_{h 1}\right]<d\left[\theta_{h}\right]+d\left[\phi_{h}\right]$. On the other hand $d\left[\theta_{h}\right]$ $\leqq d\left[\theta_{i_{j}}\right]$ so no $d\left[\theta_{i}\right]+d\left[\phi_{i}\right]$ is increased. Hence the minimality of the set $\left\{\theta_{i} \phi_{i}\right\}$ would be violated. 
Consider a particular range of indices, say $c<i \leqq c+k+1$. A term $w$ is said to be cancelled in the range if it is bound in $\theta_{c+1} \phi_{c+1}$, say $w=u^{\prime} y_{h} x_{h} v^{\prime}$ with $u^{\prime} y_{1} \in \theta_{c+1}$ and $x_{1} v^{\prime} \in \phi_{c+1}$, and if $u^{\prime} y_{h} \in \theta_{c+h}, x_{h} v^{\prime} \in \phi_{c+h}$. If all terms bound to this reaction are cancelled, that is, if $u^{\prime} y_{i} \in \theta_{c+i}, x_{i} v^{\prime} \in \phi_{c+i}$ for all $i$ in the range, then $w$ is said to be completely cancelled in the range. For the following lemma we will assume that class $A$ is nonempty so there exists some positive integer $a$ for which $d\left[\theta_{i} \phi_{i}\right]=N$ if and only if $i \leqq a$.

LEMMA 9. For some integer $p$ we have $a=p(k+1)$ and in each of the ranges $(j-1)(k+1)<i \leqq j(k+1), j=1, \cdots, p$, all terms of $\bar{\theta}_{i} \bar{\phi}_{i}$ are completely cancelled.

Proof. We proceed by induction, assuming that for some $p^{\prime} \leqq p$ (possibly 0 ) ranges for $j=1, \cdots, p^{\prime}$ have been established in which the products of the first $k_{j}$ terms of $\bar{\theta}_{i}$ by all terms of $\bar{\phi}_{i}$ are completely cancelled in the range. Remark that this means that when $i=(j-1)(k+1)+h$, all terms of $\bar{\phi}_{i}$ begin with $x_{h}$. Also a term $x_{1} v^{\prime} \in \Phi_{i}$ for $i=(j-1)(k+1)+1$ if and only if $x_{h} v^{\prime} \in \Phi_{i}$ for $i=(j-1)(k+1)+h$ for all $h=1, \cdots, k+1$. Similarly for the first $k_{j}$ terms of the corresponding $\left\{\bar{\theta}_{i}\right\}$. We assume, finally, that no term appearing in this induction schema appears anywhere else in any $\theta_{i} \phi_{i}$.

Note that if $p^{\prime}=0$, the induction hypothesis is satisfied vacuously, and if we can show that either $p^{\prime}$ or one of the $k_{j}$ can be increased, the induction is complete.

Now consider the set of all terms of degree $N$ of the $\left\{\theta_{i} \phi_{i}\right\}$ not in the induction schema, and let $w$ be the leading term of this set. If $w$ is completely cancelled in one of the already established ranges then, since it leads all terms not in the schema, it must be produced by the next succeeding term of the $\bar{\theta}_{i}$ for $i$ in the range; that is, the $k_{j}$ of this range may be increased by 1 . If $w$ should be completely cancelled in a new range, then since $\theta_{i+1} \phi_{i+1}<\theta_{i} \phi_{i}$ for all $i$, this must be a range immediately succeeding $p^{\prime}(k+1)$. It will be shown later that in this case the remaining terms of $\bar{\phi}_{i}$ in this new range also satisfy the required conditions, and hence $p^{\prime}$ would be increased by 1 . Finally, if $w$ (and the other new terms included) appear nowhere else, the induction is complete.

It will therefore be assumed that $w$ is not completely cancelled everywhere, and we agree to ignore those ranges in which it is completely cancelled. We further assume, as a first step, that $w$ is either not cancelled in an established range or appears elsewhere, and again we agree to ignore ranges (if any exist) in which $w$ is cancelled. Now $w$ cannot occur twice in the same $\theta_{i} \phi_{i}$ (for example, if $w=u^{\prime} y_{2} x_{2} v^{\prime}$ with $u^{\prime} y_{1}+u^{\prime} y_{2} \in \theta_{i}$ and $x_{1} v^{\prime}+x_{2} v^{\prime} \in \phi_{i}$, then there is an uncancelled term which exceeds $w)$. Thus since $\sum_{1}^{q} \theta_{i} \phi_{i}=1$, w must appear in two members of $\left\{\theta_{i} \phi_{i}\right\}$, say $i=h$ and $j$. Note that since $w$ has maximal degree $h, j \leqq a$.

Write $a^{\prime}=p^{\prime}(k+1)$ and consider first the case $d\left[\theta_{j}\right]<d\left[\theta_{h}\right]$ and $j>a^{\prime}$. Since the induction schema ends at $a^{\prime}, w$ must be the leading term of $\theta_{j} \phi_{j}$, 
Also since $d[w]=d\left[\theta_{h} \phi_{h}\right], w$ equals or is bound to $u v$, where $d[u]=d\left[\theta_{h}\right]$ $>d\left[\theta_{j}\right]$. Thus Lemma 5 would be violated. In fact, by Note 2, none of the terms of $u \Phi_{h}$ could be in $\theta_{j} \phi_{j}$.

If $d\left[\theta_{j}\right]<d\left[\theta_{h}\right]$ where $j \leqq a^{\prime}$ then $j$ occurs in one of the established ranges, say $(r-1)(k+1)<j \leqq r(k+1)$. By hypothesis, the first $k_{r}$ terms of each $\theta_{i}$ in this range are completely cancelled. Thus writing $\theta_{i}^{\prime}$ for $\theta_{i}$ with these terms removed, $w$ must be the leading term of $\theta_{j}^{\prime} \phi_{j}$. Also, all terms of $\bar{\phi}_{j}$ have the same initial symbol and so by Lemma 3 , if $v_{j}$ is the leading term of $\phi_{j}$, then $w$ is the leading term of $\theta_{j}^{\prime} v_{j}$. Now $w$ is of maximal degree and since all terms of $\bar{\phi}_{i}$ for $i=(r-1)(k+1)+h$ begin with $x_{h}$, it is clear that $w$ could appear in some other $\theta_{i} \phi_{i}$ of the range only if $w$ were cancelled in the range. Hence all terms of $\theta_{i}^{\prime} \phi_{i}<w$ for $i \neq h$ and $w$ is the leading term of $\sum \theta_{i} v_{i}$ where $\left\{v_{i}\right\}$ are the leading terms of the respective $\left\{\phi_{i}\right\}$ and the sum is over the range. Thus if $w$ were to equal or be bound to $u v \in \theta_{h} \phi_{h}$ then $d\left[v_{j}\right]>d[v]$ so Lemma 7 would be violated. Remark, also, that by Note 3 , none of the terms of $u \bar{\phi}_{h}$ would be in $\sum \theta_{i} \phi_{i}$.

We conclude that whenever $w \in \theta_{j} \phi_{j}, d\left[\theta_{j}\right]=d\left[\theta_{h}\right]$. Suppose, first, that $h>a^{\prime}$ so as before $w$ is the leading term of $\theta_{h} \phi_{h}$. We consider first the exceptional case: $\theta_{h}$ has leading terms $u^{\prime} y_{1}+u^{\prime} y_{2}$ and all terms of $\phi_{h}$ begin with $x_{1}$. Thus $w=u^{\prime} y_{2} x_{1} v^{\prime}$ where $x_{1} v^{\prime}$ is the leading term of $\phi_{h}$. By the minimality of the set $\left\{\theta_{i} \phi_{i}\right\}$ it is clear that $u^{\prime} y_{1} \notin \theta_{i}$ for any $i$, since otherwise such a $\theta_{i}$ could be diminished by using $\theta_{h}$, while at the same time (because $d\left[\theta_{h}\right]+d\left[\phi_{h}\right]=N$ ) $d\left[\phi_{h}\right]$ would not be increased. Similarly $u^{\prime} y_{2} \notin \theta_{i}$ for $i>a^{\prime}$ and $i \neq h$, for it would necessarily be the leading term of such $\theta_{i}$ and hence $\theta_{h}$ could be diminished. We have therefore to consider only those $\theta_{r}$ with $r \leqq a^{\prime}$ such that $u^{\prime} y_{2} \in \theta_{r}$.

Note 4. An argument similar to that of this paragraph will be used several times, and will be called the argument of Note 4 . The terms of $u^{\prime} y_{2} \phi_{h}$ are leading terms of $\theta_{h} z$ for the various terms $z$ of $\bar{\phi}_{h}$, so by Lemma 5 could not appear in any $\theta_{i} \phi_{i}$ for which $d\left[\theta_{h}\right]<d\left[\theta_{i}\right]$. Similarly, as in the discussion above, the terms of $u^{\prime} y_{2} \bar{\phi}_{r}$ are the leading terms of $\sum \theta_{i} z_{i}$ for the various sets $\left\{z_{i}\right\}$ of corresponding terms of the $\bar{\phi}_{i}$ for $i$ in the range in which $r$ occurs. Thus, by Lemma 7 , these terms also cannot occur in $\theta_{i} \phi_{i}$ for which $d\left[\theta_{r}\right]\left(=d\left[\theta_{h}\right]\right)$ $<d\left[\theta_{i}\right]$. Further, by Notes 2 and 3 , none of these terms can occur in $\theta_{i} \phi_{i}$ for which $d\left[\theta_{i}\right]<d\left[\theta_{h}\right]$. Thus terms beginning with $u^{\prime} y_{2}$ can appear only in those $\theta_{r} \phi_{r}$ for which $d\left[\theta_{r}\right]=d\left[\theta_{h}\right]$. It follows that $u^{\prime} y_{2} \bar{\phi}_{h}+\sum u^{\prime} y_{2} \bar{\phi}_{r}=0$, whence $\bar{\phi}_{h}+\sum \bar{\phi}_{r}=0$, violating Lemma 8.

The exceptional case is therefore impossible, and so $w$ is produced by the leading term of $\theta_{h}$. If $w$ were unbound throughout the whole range $1 \leqq i \leqq a$, then we would have $w=u v$ where $u$ leads in $\theta_{h}$ and $u \in \theta_{j}$. But then $\theta_{j}$ could be diminished, violating minimality. Thus $w$ must be bound somewhere, and since it is the leading term not in the induction schema, it has form $w=u^{\prime} y_{2} x_{2} v^{\prime}$. Suppose, first, that $w$ still remains unbound in $\theta_{h} \phi_{h}$, so $u^{\prime} y_{2}$ and $x_{2} v^{\prime}$ are the 
leading terms of $\theta_{h}, \phi_{h}$ respectively. By minimality, $u^{\prime} y_{2} \notin \theta_{i}$ for any $i$, and so the only other $\theta_{i} \phi_{i}$ in which $w$ appears are those in which it is bound. Thus we need consider only those $\theta_{r}$ for which $u^{\prime} y_{1} \in \theta_{r}$. If one of these $r \leqq a^{\prime}$ then all must be, for if some $r>a^{\prime}, u^{\prime} y_{1}$ would be the leading term of that $\theta_{r}$ and the remaining $\theta_{r}$ could be diminished. Thus suppose all such $r \leqq a^{\prime}$. Now all these $r$ must necessarily be the first members of the ranges in which they occur. We may then use the argument of Note 4 on the terms of $u^{\prime} y_{2} \bar{\phi}_{h}$ and $u^{\prime} y_{1} \bar{\phi}_{r}$, the set violating Lemma 8 being $\left\{\bar{\phi}_{h}, \bar{\phi}_{r+1}\right\}$ (recall that $x_{1} v^{\prime} \in \bar{\phi}_{r}$ if and only if $\left.x_{2} v^{\prime} \in \phi_{r+1}\right)$.

The remaining possibility is that $u^{\prime} y_{1}$ leads in $\theta_{j}$ for $j>a^{\prime}$. But then clearly no term of $u^{\prime} y_{2} \bar{\phi}_{h}$ or of $u^{\prime} y_{1} \bar{\phi}_{j}$ appears elsewhere (using the first part of the Note 4 argument), so all terms of $\bar{\phi}_{h}$ are of form $x_{2} v^{\prime}$ with $x_{1} v^{\prime} \in \bar{\phi}_{j}$ and conversely. Since $\theta_{i+1} \phi_{i+1}<\theta_{i} \phi_{i}$ for all $i$, we must have $j=a^{\prime}+1$ and $h=a^{\prime}+2$, that is, the first two members of a new range have been formed.

Now if $w$ is bound in $\theta_{h} \phi_{h}$, the situation is similar, for then $u^{\prime} y_{1}$ leads in $\theta_{h}$, and all terms of $\phi_{h}$ are of form $x_{1} v^{\prime}$. Thus either $u^{\prime} y_{2}$ leads in $\theta_{j}$ with $j>a^{\prime}$, so the situation is precisely as that above, with the roles of $h$ and $j$ reversed, or $u^{\prime} y_{2} \in \theta_{r}$ for certain $r \leqq a^{\prime}$. Such $r$ must be second members of their ranges and again the Note 4 argument may be used, the set violating Lemma 8 being $\left\{\bar{\phi}_{h}, \bar{\phi}_{r-1}\right\}$.

This disposes of all cases when $h>a^{\prime}$, so let $h \leqq a^{\prime}$ and $w \notin \theta_{i} \phi_{i}$ for any $i>a^{\prime}$. As in the above, the Note 4 argument shows that $w$ must be bound in some $\theta_{i} \phi_{i}$, so $w$ has form $w=u^{\prime} y_{2} x_{2} v^{\prime}$. Thus $u^{\prime} y_{1} \in \theta_{r}$ for certain $r$ which are first members of their ranges, and we may also have $u^{\prime} y_{2} \in \theta_{r^{\prime}}$ for certain $r^{\prime}$. But then, again, the Note 4 argument applies, the set violating Lemma 8 being $\left\{\Phi_{r+1}, \Phi_{r^{\prime}}\right\}$. The conclusion is that all terms beginning with $u^{\prime} y_{2}$ (including $w)$ are cancelled in the ranges in which they occur, or in the first two members of a new range.

Now assume that all terms beginning with $u^{\prime} y_{i}, i=1, \cdots, m-1$, are cancelled in the ranges in which they occur or in the first $m-1$ members of a new range, but that a term beginning with $u^{\prime} y_{m}$ is not. Such terms may occur in the following ways:

(i) $u^{\prime} y_{i} \in \theta_{c+i}$ for $i=1, \cdots, m-1$ the first $m-1$ members of a range, but $u^{\prime} y_{m} \notin \theta_{c+m}$;

(ii) $u^{\prime} y_{m} \in \theta_{j}$ for some $j \leqq a^{\prime}$;

(iii) $u^{\prime} y_{i} \in \theta_{a^{\prime}+i}, i=1, \cdots, m-1$ (that is, the first $m-1$ members of a new range have been formed);

(iv) $u^{\prime} y_{m} \in \theta_{h}$ for some $h>a^{\prime}$.

Note that since the terms beginning with $u^{\prime} y_{i}$ are leading terms not in the induction schema, in (iii) and (iv) the $u^{\prime} y_{i}$ is the leading term of $\theta_{a^{\prime}+i}$, or of $\theta_{h}$. Thus the minimality of the $\left\{\theta_{i} \phi_{i}\right\}$ assures that (iii) implies (i) is empty and (iv) implies (ii) is empty. It is thus clear that the Note 4 argument applies, 
first to show that all terms beginning with $u^{\prime} y_{m}$ are bound in some $\theta_{i} \phi_{i}$, the set violating Lemma 8 being the $\phi_{j}$ for those $j$ in which $u^{\prime} y_{m} \in \theta_{j}$ and the terms beginning with $u^{\prime} y_{m}$ unbound anywhere. Thus the $j$ of (ii) are the $m$ th members of certain ranges. The Note 4 argument then applies to (i) and (iv), the set violating Lemma 8 being $\left\{\bar{\phi}_{h}, \bar{\phi}_{c+m}\right\}$ for the $c$ of the various ranges of (i); it also applies to (ii) and (iii), the set being $\left\{\Phi_{a^{\prime}+1}, \Phi_{j-m+1}\right\}$ for the various $j$ of (ii); (i) and (ii), $\left\{\Phi_{c+m}, \phi_{j}\right\}$. There remains only the possibility (iii) and (iv) in which case, as above $h=a^{\prime}+m$ becomes the $m$ th member of a new range. It is thus clear that all terms beginning with $u^{\prime} y_{i}, i=1, \cdots$, $k+1$, are cancelled in a new, or an established range and hence, as in the earlier discussion, the induction is complete.

This disposes of all terms of degree $N$ and we turn our attention to terms of degree $N-1$. Note that secondary reaction terms are of degree $\leqq N-2$, so still do not have to be considered. Terms of degree $N-1$ may arise in the following ways: I. In $\bar{\theta}_{i} \bar{\phi}_{i}^{*}$ for class A products. Ia. In $\bar{\theta}_{i} \bar{\phi}_{i}^{*}$ for class B products. II. In $\bar{\theta}^{*}{ }_{i} \bar{\phi}_{i}$ for class A products. IIa. In $\bar{\theta}_{i}^{*} \bar{\phi}_{i}$ for class B products. III. In $\bar{\theta}_{i} \bar{\phi}_{i}$ for class $C$ products. Note that for types I and II, $i \leqq a$, and that a term cannot be both type I and II in a range, say $c+i$, $i=1, \cdots, k+1$, of Lemma 9 , for such a term would be at the same time of form $u^{\prime} x_{j} v^{\prime}$ and $u^{\prime} y_{j} v^{\prime}$.

The following lemmas will be needed:

LEMMA 10. Suppose $w$ is of type IIa or III in $\theta_{h} \phi_{h}$ and is the leading term of $\theta_{h} v_{2}$ for some $v_{2} \in \Phi_{h}$, if wequals or is bound to uv, where $d\left[v_{2}\right]>d[v]$, then for all $r$ either $u \notin \theta_{\text {r }}$ or $v \notin \phi_{r}$.

Proof. Suppose the contrary. By Lemma 5 this could not happen if $w$ is of type II, IIa, or III in $\theta_{r} \phi_{r}$. Thus assume it is of type I or Ia. If it is of type Ia, then $u \in \bar{\theta}_{r}$ for some $r$ for which $\theta_{r} \phi_{r}$ is of class B and so $u$ ends in some $x_{j}$. By Lemma $4, u$ is the leading term of $\theta_{h} z$ for some $z$, so that $z$ must also end in some $x_{j}$. Thus the elementary transformation $\theta_{r} \rightarrow \theta_{r}+\theta_{h} z, \phi_{h} \rightarrow \phi_{h}$ $+z \phi_{r}$ deletes $u$ from $\theta_{r}$ and adds to $\phi_{h}$ a term of degree $d[z]+d\left[\phi_{r}\right]-1$ $=d\left[v_{2}\right]-d[v]+d\left[\phi_{r}\right]-1$. But $d\left[v_{2}\right]=d\left[\phi_{h}\right]$ while $d[v]=d\left[\phi_{r}\right]-1$, so that $d\left[\phi_{h}\right]$ is not increased, and minimality is violated.

Now suppose $w$ is of type I in $\theta_{r} \phi_{r}$, then $u \in \bar{\theta}_{r}$ for $r$ a member of one of the ranges of Lemma 9 , say $d+j, j=1, \cdots, k+1$. Thus each $\bar{\theta}_{d+j}$ of the range has a term differing from $u$ only in its last symbol. Again, by Lemma $4, u$ is the leading term of $\theta_{h} z$, so if $u^{\prime} y_{j}$ is the term of $\bar{\theta}_{d+j}$ corresponding to $u$, then $u^{\prime} y_{j}$ is the leading term of $\theta_{h} z^{\prime} y_{j}$, where $z^{\prime} y_{j}$ differs from $z$ only in its last symbol. Thus consider the elementary transformation

$$
\begin{aligned}
\theta_{d+j} & \rightarrow \theta_{d+j}+\theta_{h} z^{\prime} y_{j}, \\
\phi_{h} & \rightarrow \phi_{h}+\sum z^{\prime} y_{j} \phi_{d+j} .
\end{aligned}
$$

Recall that form Lemma 9, $\sum y_{j} \bar{\phi}_{d+j}=0$, so 


$$
\begin{aligned}
d\left[\sum z^{\prime} y_{j} \phi_{d+j}\right] & =d\left[z^{\prime} y_{j}\right]+d\left[\phi_{d+j}\right]-1 \\
& =d[z]+d\left[\phi_{r}\right]-1 .
\end{aligned}
$$

Thus again the term added to $\phi_{h}$ has degree equal to $d\left[\phi_{h}\right]$ and since each of the $\theta_{d+j}$ is diminished, minimality is again violated.

Lemma 11. Suppose that $x_{i} v^{\prime} \in \Phi_{c+i}$ for $\{c+i\}, i=1, \cdots, k+1$ one of the ranges of Lemma 9. If $w$ is the leading term of $\sum \theta_{c+i} x_{i} v^{\prime}$ (where $w$ is in a type II or type III product) and equals $u v$, where $d\left[x_{i} v^{\prime}\right]>d[v]$, then for all $r$, either

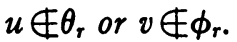

Proof. Suppose the contrary. Note that as in the remark following Lemma 6 , in this situation we could not have $u v$ a reaction to which $w$ was bound. By Lemma 6, $u=\sum \theta_{c+i} z_{i}$ for some set $\left\{z_{i}\right\}$. Clearly either $d[v]=d\left[\phi_{r}\right]$ or $d[v]=d\left[\phi_{r}\right]-1$. But in the former case, since $d\left[x_{i} v^{\prime}\right]=d\left[\phi_{c+i}\right]$, we have $d\left[v^{\prime}\right]-d[v]<d\left[\phi_{c+i}\right]-d\left[\phi_{r}\right]$, so Lemma 7 is violated. Thus $w$ is of type I or Ia in $\theta_{r} \phi_{r}$. But $w$ cannot be of type I for if $r \leqq a$ all terms of $\bar{\theta}_{r}$ end in some $y_{j}$, whereas $z_{i}$ ends in some $x_{j}$. Suppose $w$ is of type Ia in $\theta_{r} \phi_{r}$. Consider the transformation $\theta_{r} \rightarrow \theta_{r}+\sum \theta_{c+i} z_{i}, \phi_{c+i} \rightarrow \phi_{c+i}+z_{i} \phi_{r}$. Since $z_{i}$ ends in some $x_{j}$ we have $d\left[z_{i} \phi_{r}\right]=d\left[z_{i}\right]+d\left[\phi_{r}\right]-1=d\left[x_{i} v^{\prime}\right]-d[v]+d[v]=d\left[\phi_{c+i}\right]$. Thus the transformation violates minimality.

Remark that in the following lemma we will need to use an argument similar to that of Note 4. The only difference will be that Lemmas 10 and 11 will be needed in certain cases. These will be: Lemma 11 to show that a term of type II or III which leads in $\sum \theta_{c+i} x_{i} v^{\prime}$ is not of type I or Ia in any $\theta_{r} \phi_{r}$ for which $d\left[\phi_{r}\right]<d\left[\phi_{c+i}\right]$; Lemma 10 to show that a term of type IIa or III which leads in $\theta_{h} v_{2}$ is not of type I or Ia in $\theta_{r} \phi_{r}$ for which $d\left[\phi_{r}\right]<d\left[\phi_{h}\right]$. However, we will continue to call this the argument of Note 4.

LEMMA 12. (i) In the ranges established by Lemma 9 , all terms of $\bar{\theta}_{i} \bar{\phi}_{i}^{*}$ and $\bar{\theta}_{i}^{*} \bar{\phi}_{i}$ are completely cancelled. (ii) No class B products exist. (iii) If $b$ is an integer such that $\theta_{i} \phi_{i}$ is of class $\mathrm{C}$ if and only if $a<i \leqq b$, then $b=d(k+1)$ for some integer $d$, and for such $i$, additional ranges exist in which all terms of $\bar{\theta}_{i} \bar{\phi}_{i}$ are completely cancelled.

Proof. We again set up an induction schema, assuming that for $i \leqq a$, certain leading terms of $\bar{\theta}_{i}^{*}$ and $\bar{\phi}_{i}^{*}$ follow the already established pattern; for certain $r>a, \theta_{r} \phi_{r}$ is of class $\mathrm{B}$, with certain (but not all) the terms of $\bar{\theta}_{r}^{*}$ ending in some $x_{j}$ and the leading term of $\phi_{r}^{*}$ beginning with some $x_{j}$; certain ranges $\{c+i\}, i=1, \cdots, k+1$ with $c+i>a$ have been established in which certain leading terms of $\bar{\theta}_{i}$ and all terms of $\bar{\phi}_{i}$ follow a pattern similar to that of Lemma 9.

If the induction is not complete, there exist terms of degree $N-1$, and let $w$ be the leading term not in the induction schema. As before, we agree to ignore any ranges in which $w$ is cancelled. Suppose first, that $w$ is of type I 
or Ia in $\theta_{r} \phi_{r}$. By Lemma 5 , $w$ cannot be of type I, Ia, or III in any $\theta_{j} \phi_{j}$ for which $d\left[\theta_{r}\right]<d\left[\theta_{j}\right]$, or of type II or IIa in $\theta_{j} \phi_{j}$ for which $d\left[\theta_{r}\right]<d\left[\theta_{j}\right]-1$. Also by Lemma 5 , $w$ could not be of type I or Ia in $\theta_{j} \phi_{j}$ for which $d\left[\theta_{j}\right]$ $<d\left[\theta_{r}\right]$. For type III we have the same result, by Lemma 11, when $r$ is in one of the ranges assumed in the induction hypothesis, or by Lemma 10, if it is not. Finally, Lemmas 10 and 11 show that $w$ could not be type IIa or II in $\theta_{r} \phi_{r}$ for which $d\left[\theta_{r}\right]-1<d\left[\theta_{j}\right]$.

Now if $w$ is of type Ia in $\theta_{r} \phi_{r}$ then $w=u v$ where $u$ leads in $\theta_{r}$. Since $w$ must appear in some other $\theta_{j} \phi_{j}$, it follows from the preceding paragraph that $u \in \theta_{j}$. But then $\theta_{j}$ could be diminished (without increasing $d\left[\phi_{r}\right]$ ) violating minimality. Similarly, if $w$ were of type I in $\theta_{r} \phi_{r}$ and unbound throughout. Suppose, then, that $w$ is unbound in $\theta_{r} \phi_{r}$ but bound elsewhere, so $r$ must be the second member of its range and $w=u v$ where $u$ leads in $\theta_{r}$. But then the leading term of $\theta_{r-1}$ differs from $u$ only in its final symbol. Thus $\theta_{r-1}$ and $\theta_{r}$ could diminish $\theta_{j}$ for respectively bound or unbound occurrences of $w$. The situation is clearly similar if $w$ is bound in $\theta_{r} \phi_{r}$. We conclude that $w$ is not of type Ia and is of type I only if it is cancelled in its range.

Next, consider the case $w$ is type II or IIa in $\theta_{r} \phi_{r}$. According to the preceding discussion, it cannot be type I or Ia in any $\theta_{j} \phi_{j}$. Also, by Lemmas 10 and $11 w$ cannot be type II or IIa in any $\theta_{j}$ for which $d\left[\theta_{j}\right] \neq d\left[\theta_{r}\right]$ or type III in any $\theta_{j} \phi_{j}$ for which $d\left[\theta_{j}\right] \neq d\left[\theta_{r}\right]-1$.

Now suppose $w$ is of type II in $\theta_{r} \phi_{r}$ and unbound throughout, or of type IIa, so that $w=u v$, with $u \in \bar{\theta}_{r}^{*}$ and $v \in \bar{\phi}_{r}$. From the preceding paragraph $w \in \theta_{j} \phi_{j}$ only if $u \in \theta_{j}$. Clearly all members of $u \Phi_{r}$ are unbound and also all members of $u \bar{\phi}_{j}$ for all $j$ in which $u \in \theta_{j}$, except possibly a type III case outside the ranges of the induction hypothesis. But in such a case, $u$ would be the leading term of the $\theta_{j}$ which could then be used to diminish $\theta_{h}$. We may then use the Note 4 argument, the set violating Lemma 8 being $\left\{\bar{\phi}_{r}, \bar{\phi}_{j}\right\}$ for all $j$ with $u \in \theta_{j}$.

It is now established that no type Ia or IIa products exist, that is, class $B$ products do not exist. We may therefore simplify the induction hypothesis for type III (class C) products to: if $b$ is the integer such that $\theta_{i} \phi_{i}$ is of type III if and only if $a<i \leqq b$, then for some $b^{\prime}=d^{\prime}(k+1)$ with $d^{\prime}$ an integer, the additional ranges assumed are from $a$ to $b^{\prime}$.

Returning to type II products, suppose $w$ is unbound in $\theta_{r} \phi_{r}$ but bound in some $\theta_{j} \phi_{j}$, then $w=u_{1} v_{1}$ with $u_{1} \in \bar{\theta}_{r}^{*}$ and $r$ the second member of its range. If $j>b^{\prime}$ then clearly no term beginning with $u_{1}$ could be bound in any other $\theta_{i} \phi_{i}$. Also $u_{1} \notin \theta_{i}$ for any $i>b^{\prime}$ for $u_{1}$ would lead in such $\theta_{i}$, which could then diminish $\theta_{r}$. Thus the argument of Note 4 applies, the set violating Lemma 8 being $\left\{\bar{\phi}_{r-1}, \bar{\phi}_{j}, \bar{\phi}_{i-1}\right\}$ for all $i-1$ such that $u_{1} \in \theta_{i}$. If $j \leqq b^{\prime}$ then $j$ is the first member of a range, and if $u$ is the term corresponding to $u_{1}$, it is clear that neither $u$ nor $u_{1} \in \theta_{i}$ for any $i>b^{\prime}$. Thus the argument of Note 4 again applies to $\left\{\Phi_{i}\right\}$ consisting of all $i$ such that $u \in \theta_{i}$ or $u_{1} \in \theta_{i+1}$. The discussion is similar if $w$ is bound in $\theta_{r} \phi_{r}$, for either the situation is precisely that just considered, 
or $u_{1}$ leads in some $\theta_{j}$ for $j>b^{\prime}$ while $u \in \theta_{i}$ for some $i \leqq b^{\prime}$. But again the argument of Note 4 may be applied to $\left\{\Phi_{i}, \Phi_{j}\right\}$ for all $i$ such that $u \in \theta_{i-1}$. We conclude that also for type II, $w$ can appear only if it is cancelled in the range in which it appears.

Finally, if $w$ appears only in type III products, the situation is evidently precisely that of Lemma 9. We conclude that $w$ and, in fact, if $w=u^{\prime} y_{2} x_{2} v^{\prime}$, all terms beginning with $u^{\prime} y_{2}$ are cancelled in an established range, or in the first two members of a new range.

Thus assume, again, that all terms beginning with $u^{\prime} y_{i}, i=1, \cdots, m-1$, are cancelled in some range, but that $u^{\prime} y_{m}$ is not. It is clear that a discussion similar to that of Lemma 9 will show that this is impossible. That is, if it is of type $I$ in a product, then by Lemmas 5, 7, 10, 11 it could appear only in type I or III products with equal $d\left[\theta_{j}\right]$ or in type II products with one less $d\left[\theta_{j}\right]$. But this is ruled out by minimality. If the term is of type II, then by what we have just said, it can only appear in type III or other type II products. But an argument precisely parallel to that of Lemma 9, using the argument of Note 4, shows that this is also impossible. Finally, if the term is only in type III products, the situation evidently duplicates that of Lemma 9. Thus it is seen that the induction is complete.

From the discussion of the preceding lemmas, it is clear that we have two possibilities to consider: (i) All class $\mathrm{A}$ and class $\mathrm{C}$ products are in ranges established in the manner discussed above. (ii) No products of class A, B, or C exist.

Suppose (i) is the case, and we consider the first range, $0<i \leqq k+1$. By Lemmas 9 and 12 all terms of $\theta_{i}$ of degree $\geqq d\left[\theta_{i}\right]-1$ end in $y_{i}$ and all terms of $\phi_{i}$ of degree $\geqq d\left[\phi_{i}\right]-1$ begin with $x_{i}$. Partition $\Theta$ and $\Phi$ into $\Theta=\left(\Theta_{3}, \Theta_{4}\right)$, $\Phi=\left(\Phi_{3}, \Phi_{4}\right)^{\prime}$, where $\Theta_{3}$ and $\Phi_{3}$ contain the first $k+1$ elements of $\Theta$ and $\Phi$ respectively. Form $\Theta_{1}$ and $\Phi_{1}$ from $\Theta$ and $\Phi$ by replacing $\Theta_{3}$ by $\Theta_{3} X$ and $\Phi_{3}$ by $Y \Phi_{3}$. Since $X Y=I_{k+1}$ and $Y X=1$, it is clear that $\Theta_{1} \Phi_{1}=1$ and $\Phi_{1} \Theta_{1}$ $=I_{q-k}$. Further, it is evident that $\Theta_{1}$ differs from $\Theta$ only in that its first $k+1$ elements are replaced by a single element of degree at least one less than that of the elements replaced. $\Phi_{1}$ differs from $\Phi$ in a similar fashion, and hence the minimality of the $\left\{\theta_{i} \phi_{i}\right\}$ is violated.

The other possibility, (ii), can only happen if for all $i$ such that $d\left[\theta_{i}\right]$ $+d\left[\phi_{i}\right]=N$, we have $d\left[\theta_{i} \phi_{i}\right] \leqq N-2$, and clearly no generality is lost if we assume this is true for $i=1$. This means that either all terms of $\bar{\theta}_{1}$ and $\bar{\theta}_{1}^{*}$ end with some $x_{i}$ and all terms of $\bar{\phi}_{1}$ begin with some $y_{j}$, or all terms of $\bar{\theta}_{1}$ end with some $x_{i}$ and all terms of both $\phi_{1}$ and $\phi_{1}^{*}$ begin with some $y_{j}$. Write $\Theta_{1}=\left(\theta_{1} y_{1}, \cdots, \theta_{1} y_{k+1}, \theta_{2}, \cdots, \theta_{q}\right), \Phi_{1}=\left(x_{1} \phi_{1}, \cdots, x_{k+1} \phi_{1}, \phi_{2}, \cdots, \phi_{q}\right)^{\prime}$. whence $\Theta_{1} \Phi_{1}=1$ while $\Phi_{1} \Theta_{1}=I_{q+k}$. We have thus replaced $\theta_{1}$ by $\left\{\theta_{i 1}\right\}, \phi_{1}$ by $\left\{\phi_{i 1}\right\}$, $i=1, \cdots, k+1$ with either all $d\left[\theta_{i 1}\right]<d\left[\theta_{1}\right]$ and $d\left[\phi_{i 1}\right] \leqq d\left[\phi_{1}\right]$, or all $d\left[\theta_{i 1}\right]$ $\leqq d\left[\theta_{1}\right]$ and $d\left[\phi_{i 1}\right]<d\left[\phi_{1}\right]$. Again the minimality of the $\left\{\theta_{i} \phi_{i}\right\}$ is violated. According to the discussion at the beginning of this section, we have established 
THEOREM $8\left(^{6}\right)$. There exists a ring of type $(n, k)$ for arbitrary positive integers $n, k$.

\section{THEOREM 9. There exist semi-simple rings of arbitrary type.}

Proof. There certainly exist dimensional semi-simple rings (for example, any division ring). Thus we need only show the semi-simplicity of the rings whose direct sum is the ring discussed above. However [3, p. 322] the ring of type $(n, 1), n \geqq 2$ constructed in [3] satisfies the condition $d[\alpha \beta]=d[\alpha]$ $+d[\beta]$. Thus a relation $\alpha+\beta-\alpha \beta=0$ would imply $\alpha$ or $\beta=1$, a contradiction. We thus need only consider the ring $A$ of the present section. Let $\alpha$ be a member of a right ideal $I$. If $\alpha$ is multiplied on the right by a monomial $u$ in the $\left\{y_{j}\right\}$, then for $d[u]$ sufficiently large, $\alpha u$ is a polynomial in the $\left\{y_{j}\right\}$ alone. At least one such polynomial is nonzero, for otherwise suppose $h$ is the least integer such that $\alpha u=0$ for all $u$ with $d[u]=h$. Then for some $u^{\prime}$ with $d\left[u^{\prime}\right]$ $=h-1$ we have $\alpha u^{\prime} \neq 0$, while $\alpha u^{\prime} y_{j}=0$ for all $j$. But then $\sum \alpha u^{\prime} y_{j} x_{j}=\alpha u^{\prime}=0$. Now if $\alpha$ is a polynomial in the $\left\{y_{j}\right\}$ alone, we have already seen that $d[\alpha \beta]=d[\alpha]+d[\beta]$ for any $\beta$. Thus again the relation $\alpha+\beta-\alpha \beta=0$ is impossible. Since neither summand has a right-quasi-regular right ideal, the radical is zero $\left({ }^{7}\right)$.

\section{REFERENCES}

1. C. J. Everett, Jr., Vector spaces over rings, Bull. Amer. Math. Soc. 48 (1942), 312-316*

2. W. G. Leavitt, Modules over rings of words, Proc. Amer. Math. Soc. 7 (1956), 188-193.

3. - Modules without invariant basis number, Proc. Amer. Math. Soc. 8 (1957), $322-328$. 1948.

4. N. Jacobson, The theory of rings, Math. Surveys No. 2, Amer. Math. Soc., New York,

5. J. Dieudonné, Sur le nombre de dimensions d'un module, C. R. Acad. Sci. Paris 215 (1942), 563-565.

6. W. G. Leavitt, Finite dimensional modules, An. Acad. Brasil. Ci. 27 (1957), 241-250.

7. N. Jacobson, Structure of rings, Amer. Math. Soc. Colloq. Publ. Vol. 37, Amer. Math. Soc., Providence, R. I., 1956.

8. M. P. Drazin, A generalization of polynomial identities in rings, Proc. Amer. Math. Soc. 8 (1957), 352-361.

\section{UNIVERSITY OF NEBRASKA, LINCOLN, NEBRASKA}

${ }^{(6)}$ An alternative construction exists for rings of arbitrary type $(n, k)$, proceeding directly from the polynomial ring, over the integers modulo 2 , in noncommutative symbols $\left\{x_{i j}, y_{r s}\right\}$, $i, s=1, \cdots, n+k$ and $j, r=1, \cdots, n, A$ being its quotient ring modulo the ideal generated by the elements of $X Y-I_{n+k}, Y X-I_{n}$. This ring may be handled by methods similar to those of the present section, although the discussion is somewhat more complicated. It can be shown that for all $n \geqq 2$ this ring has no zero divisors.

( ${ }^{7}$ ) For the ring mentioned in footnote 6 , it can be shown that if $I, J$ are any nonzero right ideals, there exist $\alpha \in I, \beta \in J$ with $d[\alpha \beta]=d[\alpha]+d[\beta]$. Thus the additional result: There exist prime rings of arbitrary type. 